\title{
Improved Multi-Layer Perceptron for Recognition of Control Chart Pattern
}

\author{
O. El Farissi \\ Ibn Zohr University, ENSA-A \\ BP 1136, Agadir, Morocco
}

\author{
H. Elboujaoui \\ Ibn Zohr University, ENSA-A \\ BP 1136, Agadir, Morocco
}

\begin{abstract}
This work presents a prediction of control chart pattern using a Neural Network Multilayer. A Multilayer model configuration of one hidden layer with nonlinear sigmoid activation and the Bayesian algorithm, is used. Good results with hay accuracy obtained shows that the neural network is performant to predict the control chart pattern.
\end{abstract}

\section{Keywords}

Neural Network, Multi-Layer Perceptron (MLP), Control Charts, Control Charts Pattern (CCP)

\section{INTRODUCTION}

The control chart is the commonly used tool for monitoring and surveillance of production processes.

The prediction of models of control charts becomes an objective of researchers in this field [1], [2], [3], [4], [5] ..., whose use of artificial intelligence tools, mainly neural networks, was dominant.

The models often found in control charts are: normal (NOR), upward trend (IT), downward trend (DT), upward shift (US), downward shift (DS) and cyclic (CYC), as shown in Figure 1 [1].

The NOR model indicates that the process is under control; as long as the other models indicate that the process is not under control.

Anagun [6] used a reverse propagation network (BPN) to recognize patterns in SPC. The training data was organized in two different ways: direct representation and histogram representation. The results show that the latter method provided better performance than direct representation.

In addition, Guh and Tannock [7] dealing with unique patterns such as sudden changes, linear trends or cyclical patterns, studied the use of a (BPN) to recognize patterns of simultaneous control graphs when multiple patterns exist together.

N.V.N. Kiran et al. [8] assessed the relative performance of the five learning algorithms. The structures of the CCP Recognizer tested in this study include an input layer, a hidden layer and an output layer. The best result is formed with a traindx algorithm.

This article presents an improvement of the MLP model which leads to a good recognition of the control chart models.

The rest of the paper is organized as follow: The second section will review the use of Multilayer Network. The third section will present the Neural Network for Pattern Recognition as fellow: Data collection, neural network design for pattern recognition and discussion of the results.

\section{MULTILAYER NETWORK}

Artificial Neural Network is a model composed by lot of neurons connected with each other through parameters called weights. The neuron is a mathematical function that receive a linear combination of inputs parameters by weights and produce an output (Figure 1).

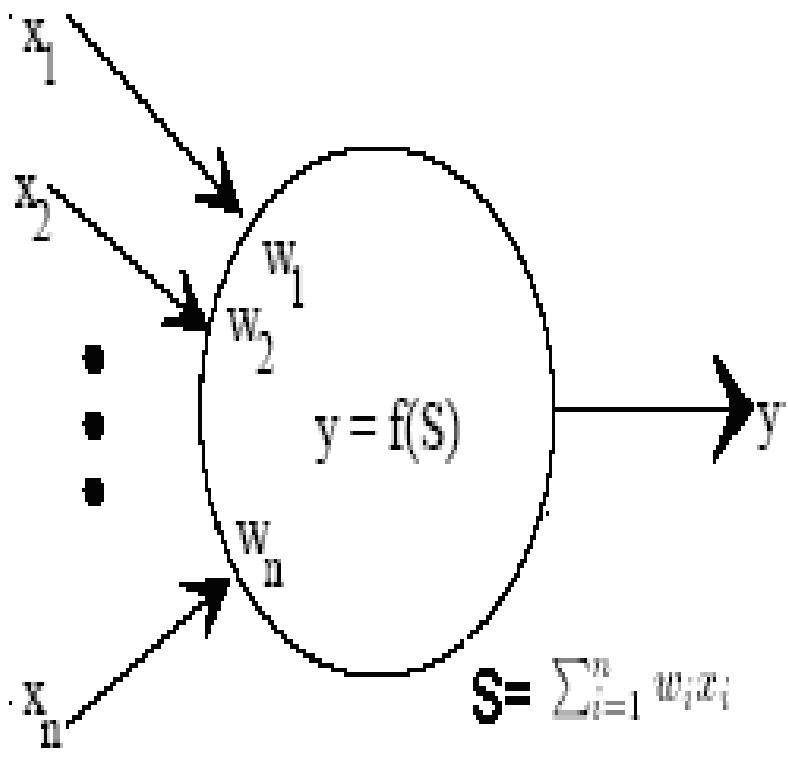

Fig 1: A formal neuron

The output of the neuron is given by $\left.\right|_{i}-j\left(\sum_{i=1}^{n} w_{j} x_{i}\right\rangle$ where $x-\left\{X_{1}, Y_{2}, \ldots, Y_{y_{i}}\right\rangle$ is the input vector and $\left\langle\left(w_{j}\right\rangle\right.$ are the weights. The activation function $f$ depends on the type of problem to be solved by the network.

The architecture of the neural network is defined by the manner that the neurons are connected between them. The important architecture of neural network is the Multi-Layer Perceptron (Figure 2) which is most used in classification and regression problems [9], [10], [11].

Supervised learning is the important learning technique most used in order to train and determine the network parameters through input output data. Supervised learning proceeds in the following steps: 


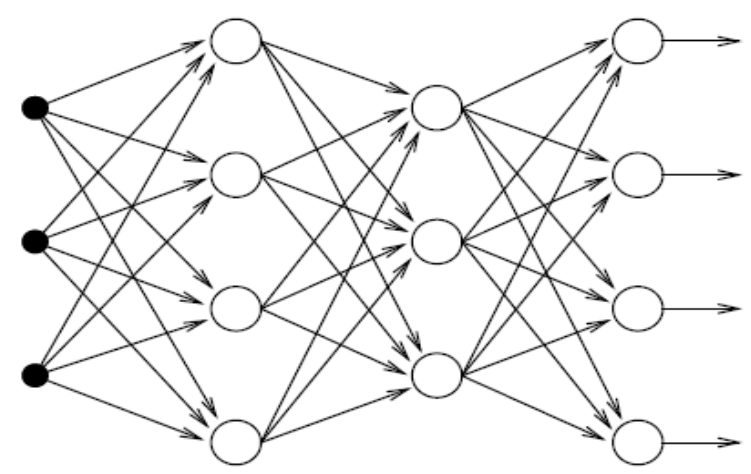

Fig 2 : Multi-Layer Perceptron. From left to right: input layer, first hidden layer, second hidden layer, output layer

1. Initialize weights

2. Enter input into the neural network and obtain the output.

3. Compute the error between output of the and the correct output.

4. Adjust the weights to reduce the error.

5. Repeat steps 2 - 4 for all training data.

\section{NEURAL NETWORK FOR PATTERN RECOGNITION}

\subsection{Data collection}

There are six main types of patterns that commonly appear in CCPs (Figure 3): normal (NOR), increasing trend (IT), decreasing trend (DT), upward shift (US), downward shift (DS) and cyclic (CYC).

Since real process containing all type of patterns is not available, simulated data are often used [8], [12]. Table 1 describe the equations used to generate the data points for the each pattern.

Table 1. Generated data for various patterns

\begin{tabular}{|c|c|}
\hline Patterns & Equations \\
\hline Normal & (1) \\
\hline $\begin{array}{c}\text { Trend } \\
\text { incresing }\end{array}$ & 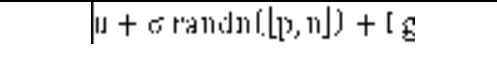 \\
\hline $\begin{array}{c}\text { Trend } \\
\text { decresing }\end{array}$ & 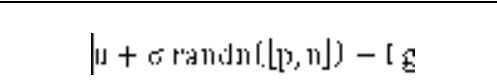 \\
\hline Shift up & 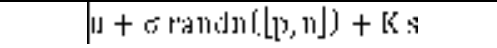 \\
\hline Shift down & 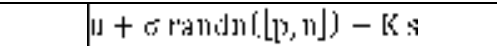 \\
\hline Cyclic & 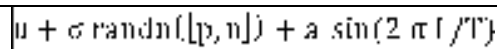 \\
\hline
\end{tabular}

\subsection{Neural network design}

In this study the MLP used consist of an input layer, one hidden layer and an output layer and (Figure 4). In input layer, the $\mathrm{n}$ nodes correspond to the sample size used for process control. A single neuron was required for output layer with the normalized coding shown in Table 2.

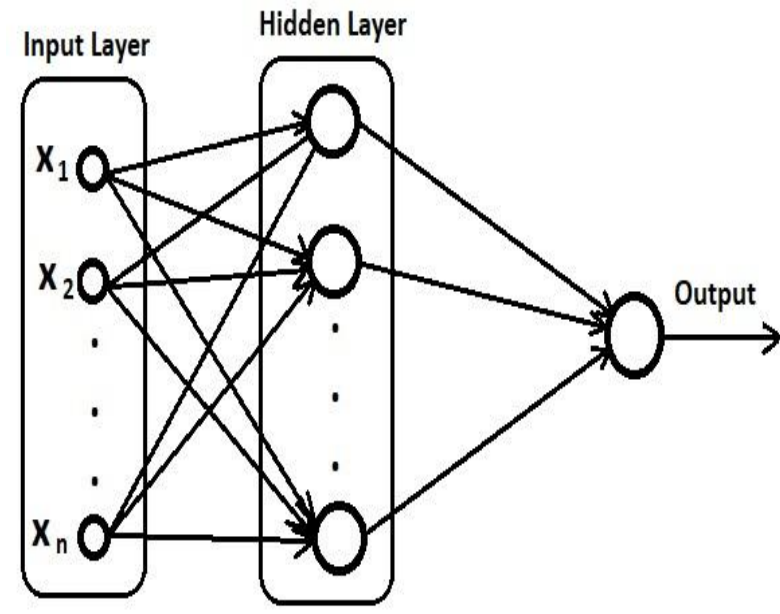

Fig 4: The structure of MLP used for CCPs

For each type of the six CCPs, $1 \mathrm{t} \times \mathrm{r}$ samples data were generated using the values of parameters shown in Table 2

Table 2. Parameters and output for Each Pattern

\begin{tabular}{|c|c|c|}
\hline Patterns & \multicolumn{1}{|c|}{$\begin{array}{c}\text { Parameter's } \\
\text { values }\end{array}$} & output \\
\hline Normal & $\mu=0, \sigma=1$ & 0.5 \\
\hline Cyclic & $\mathrm{a}=25, \mathrm{~T}=\mathrm{np}$ & -0.5 \\
\hline Increasing Trend & $\mathrm{g}=0.3$ & 0.1 \\
\hline Decreasing Trend & $\mathrm{g}=0.3$ & -0.1 \\
\hline Shift Up & $\mathrm{s}=4$ & 0.9 \\
\hline Shift down & $\mathrm{s}=4$ & -0.9 \\
\hline
\end{tabular}

\subsection{Tests and results}

In order to train the network, the three most used algorithms Multi-Layer Perceptron are considered: Levenberg-Marquardt algorithm (trainlm), Bayesian Regularization (trainbr) and Scaled Conjugate Gradient (trainscg). the number of neurons in hidden layer is adjusted in order to determine the efficient architecture of the problem.

Table 3 compare the correlation coefficient between outputs and correct outputs for each training algorithm and different numbers of neurons in hidden layer. The best correlation coefficient is obtained for trainbr algorithm with 8 neurons in hidden layer.

Table 3. Comparison Of The Coefficient Of Correlation

\begin{tabular}{|c|c|c|c|}
\hline NNHL & trainlm & trainbr & trainscg \\
\hline 7 & 0.99511 & 0.99865 & 0.96834 \\
\hline 8 & 0.99886 & 1 & 0.97494 \\
\hline 9 & 0.9869 & 0.99629 & 0.94187 \\
\hline 10 & 0.99325 & 0.99982 & 0.95493 \\
\hline 11 & 0.99888 & 0.99996 & 0.97736 \\
\hline 8 & 0.96938 & 0.99356 & 0.95089 \\
\hline 13 & 0.99974 & 0.99999 & 0.98164 \\
\hline
\end{tabular}




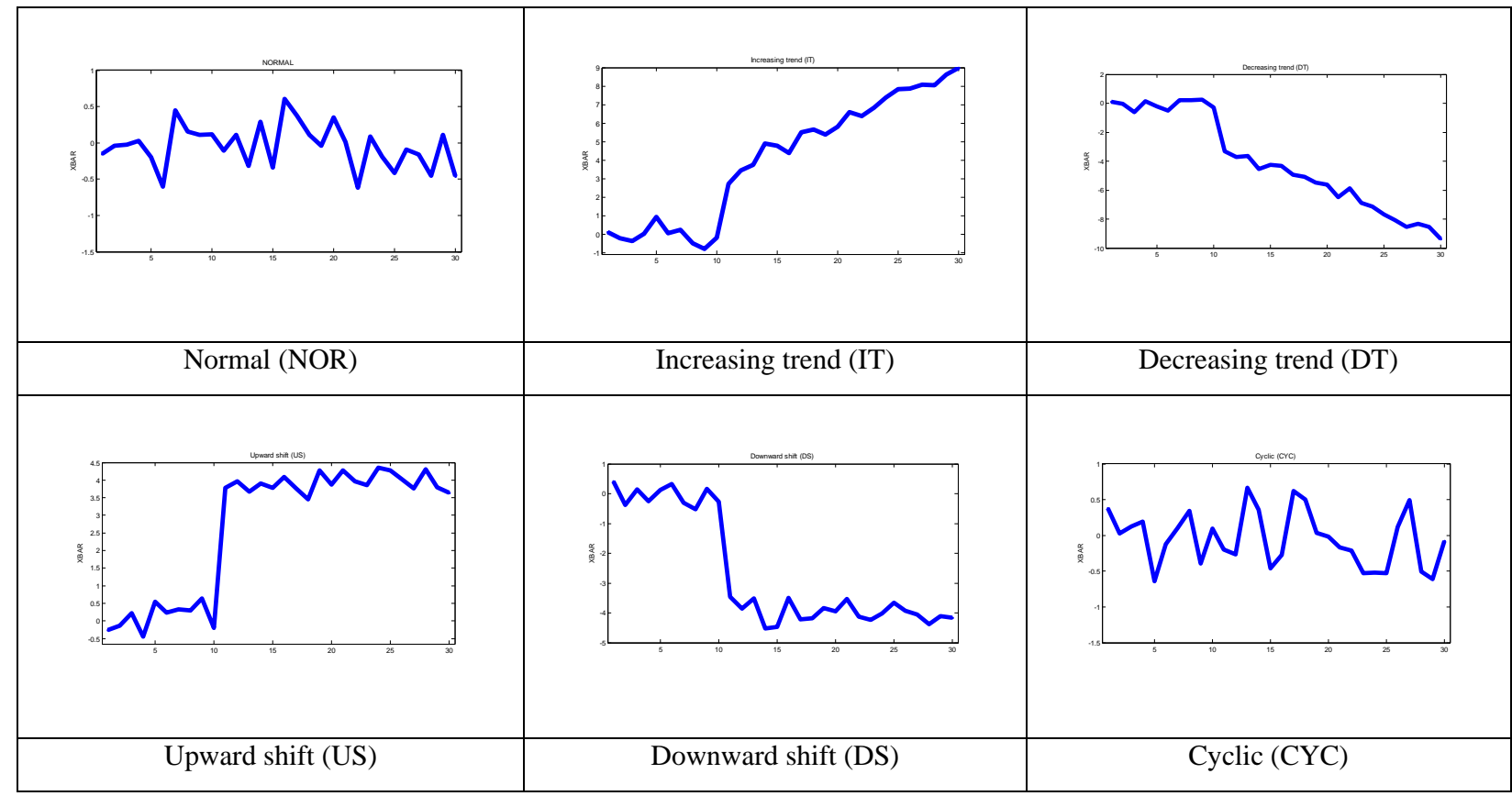

Fig 3: Six types of basic Control Charts Pattern [1]

The comparison of the mean sum of squares of the network errors (MSE) in Table 4 shows also that trainbr algorithm with 8 neurons in hidden layer is the best architecture to control chart pattern recognition.

Table 4. Comparison of The MSE Performance

\begin{tabular}{|c|c|c|c|}
\hline NNHL & trainlm & trainbr & trainscg \\
\hline 7 & $5.3077 \mathrm{e}-04$ & $7.9369 \mathrm{e}-04$ & 0.0149 \\
\hline 8 & 0.0012 & $3.3889 \mathrm{e}-09$ & 0.0215 \\
\hline 9 & 0.0016 & 0.0022 & 0.0201 \\
\hline 10 & 0.0028 & $1.0796 \mathrm{e}-04$ & 0.0248 \\
\hline 11 & $7.0253 \mathrm{e}-04$ & $2.3320 \mathrm{e}-05$ & 0.0166 \\
\hline 8 & 0.0024 & 0.0038 & 0.0213 \\
\hline 13 & $5.4585 \mathrm{e}-04$ & $8.1453 \mathrm{e}-06$ & 0.0141 \\
\hline
\end{tabular}

From the above results, the Multi-Layer Perceptron with 8 neurons in hidden layer and Bayesian Regularization algorithm, which is the best configuration and the most accurate architecture, is retained. In the rest of this paragraph, more details on the results obtained with the selected architecture are given.

Figure 5 describe the training and validation errors. In this figure, the final mean-square error is very small and the neural network generalize very well, and no overfitting has occurred. The predictive model gives very good predictions on the data of the training and testing data.

The results of a linear regression between the outputs and the corresponding targets are shown in Figure 6. The output of the neural network tracks the targets very well for both of training and testing data, and the correlation coefficient value is 1 for the total response.

Finally, in Figure 7 the absolute errors between targets and Outputs of neural network is presented. it's remarkable that they are practically equal to zero in the majority of cases. The maximum error is detected in observation 662 and is equal to 0.0017 .

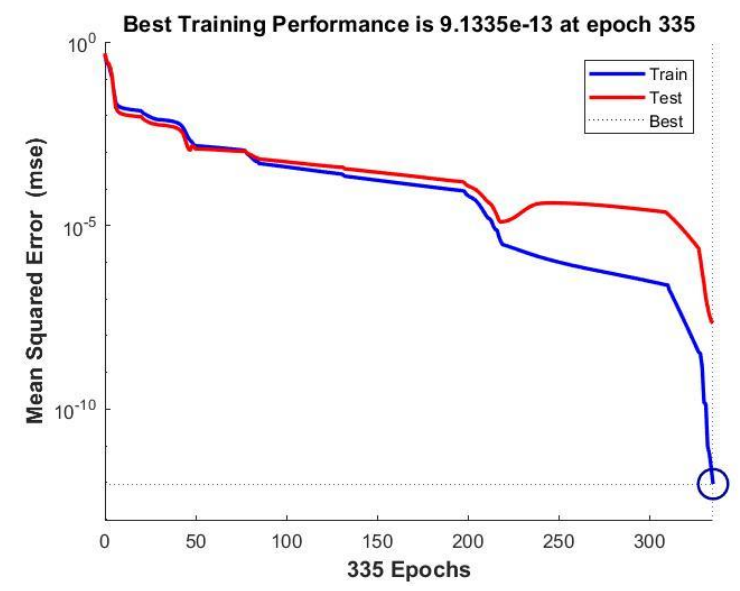

Fig 5: Mean squared error for training and testing data

Table 5 compare the use of neural network design for recognition of control chart pattern with the work [1].

Table 5. Generated data for various patterns

\begin{tabular}{|c|c|c|c|}
\hline $\begin{array}{c}\text { Architecture } \\
\text { of MLP }\end{array}$ & $\begin{array}{c}\text { Number of } \\
\text { Weights }\end{array}$ & Correlation & MSE \\
\hline $13 \times 13 \times 3 \times 1$ & 507 & 0.99991 & 0.00356 \\
\hline $10 \times 8 \times 1$ & 80 & 1 & $3.3889 \mathrm{e}-09$ \\
\hline
\end{tabular}

In [1], the 10 first nodes in the input layer correspond to the sample size used for process control and the remaining nodes represent the statistics of the observations. Furthermore, there are two hidden layers with 13 and 3 neurons which increases the complexity of the neural network. The number of connections between neurons in [1] is 507 .

The neural network design used in current work is much better in terms of complexity where the number of connections is 
only 80. Also, the architecture has advantages in terms of correlation and MSE.
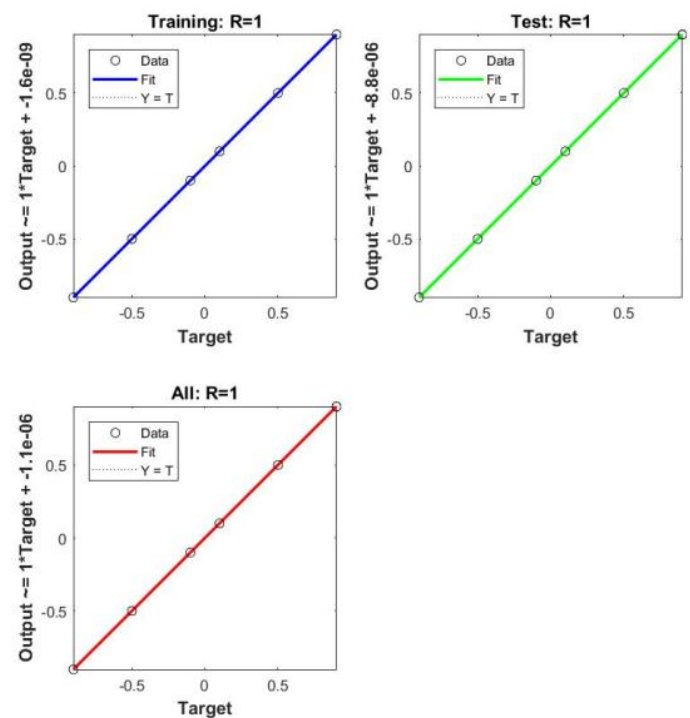

Fig 6: Neural network training regression

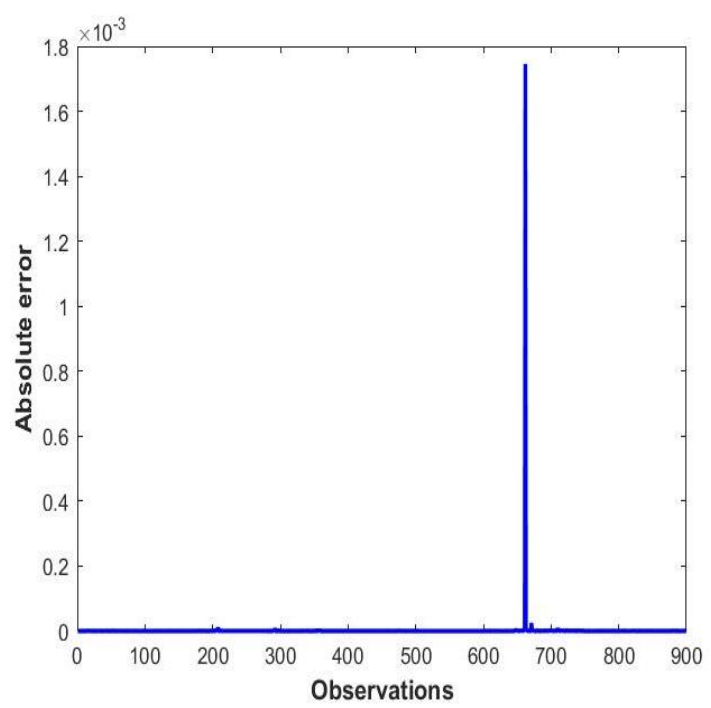

Fig 7: The absolute errors between targets and Outputs

\section{CONCLUSION}

In this article, the objective is to improve the quality of CCP recognition by an MLP network. To assess its relative performance, the architecture of the MLP was chosen after several tests of the different training structures and algorithms. The results show that an MLP with 8 neurons and a single hidden layer trained with the trianbr propagation algorithm gave better performance: a correlation coefficient equal to 1 and an MSE equal to 0.0017 .

\section{REFERENCES}

[1] O. EL FARISSI and all, Recognition Improvement of Control Chart Pattern Using Artificial Neural Networks, International Review on Modelling and Simulations (I.RE.M.O.S.), Vol. 8, N. 2 ISSN 1974-9821 April 2015.

[2] N.V.N. Indra Kiran, Effective Control Chart Pattern Recognition Using Artificial Neural Networks, IJCSNS International Journal of Computer Science and Network Security, VOL.10 No.3, March 2010.

[3] W. Laosiritaworn and T.t Bunjongjit, Classification Techniques for Control Chart Pattern Recognition: A Case of Metal Frame for Actuator Production, Chiang Mai J. Sci, 40(4): 701-712, 2013.

[4] A. D. karaoglan, An Integrated Neural Network Structure for Recognizing Autocorrelated and Trending Processes, Mathematical and Computational Applications, Vol. 16, No. 2, pp. 514-523, 2011.

[5] M. A. Hadiyat and K. R. Prilianti, Comparing Statistical Feature and Artificial Neural Networks for Control Chart Pattern Recognition: A Case Study,The 3rd International Conference on Technology and Operations Management "Sustaining Competitiveness through Green Technology Management" Bandung - Indonesia, July 4-6, 2012.

[6] A. S. Anagun, A Neural Network Applied to Pattern Recognition in Statistical Process Control, Computers \& Industrial Engineering 35, 185-188, 1998.

[7] R. S. Guh and J. D. T. Tannock, Recognition of Control Chart Concurrent Patterns Using a Neural Network Approach, International Journal of Production Research 37, 1743-1765, 1999.

[8] N.V.N. IndraKiran,M.Pramiladevi and G.Vijaya Lakshmi, Training Multilayered Perceptrons for Pattern Recognition: A Comparative Study of Five Training Algorithms, IMECS, Vol. I, March 2011.

[9] Fionn Murtagh, Multilayer perceptrons for classification and regression, Neurocomputing 1990.

[10] R. Vendrame, R. S. Braga, Y. Takahata ; and D. S. Galvão: Structure-Activity Relationship Studies of Carcinogenic Activity of Polycyclic Aromatic Hydrocarbons Using Calculated Molecular Descriptors with Principal Component Analysis and Neural Network Methods, J. Chem. Inf. Comput. Sci. 1999.

[11] E. Agirre-Basurko, G. Ibarra-Berastegi, I. Madariaga, Regression and multilayer perceptron-based models to forecast hourly $\mathrm{O}_{3}$ and $\mathrm{NO}_{2}$ levels in the Bilbao area. Environmental Modelling \& Software, 21, 4, 430-446, 2006.

[12] B. M.Wilamowski, Neural Network Architectures. Industrial Electronics Handbook (vol. 5 - Intelligent Systems, 2nd Edition, chapter 6, pp. 6-1 to 6-17, CRC Press, 2011). 